СРАВНИТЕЛЬНАЯ ОЦЕНКА ЭНЕРГЕТИЧЕСКОГО ОБМЕНА, ОСОБЕННОСТЕЙ КОМПОЗИЦИОННОГО СОСТАВА ТЕЛА И МЕТАБОЛИЧЕСКИХ НАРУШЕНИЙ У ДЕТЕЙ С ГИПОТАЛАМИЧЕСКИМ И КОНСТИТУЦИОНАЛЬНО-ЭКЗОГЕННЫМ ОЖИРЕНИЕМ

( П.Л. Окороков* , А.Л. Калинин, Н.А. Стребкова, М.А. Карева, О.В. Васюкова, В.А. Петеркова, О.Б. Безлепкина

Национальный медицинский исследовательский центр эндокринологии, Москва, Россия

Обоснование. Гипоталамическое ожирение часто развивается после лечения краниофарингиомы и характеризуется быстрым набором веса, высокой частотой метаболических нарушений, особенностями композиционного состава тела и резистентностью к стандартным подходам по модификации образа жизни и медикаментозной терапии ожирения. Недавние исследования демонстрируют, что одним из механизмов, объясняющих повышенную прибавку веса у таких детей, является снижение основного обмена (ОО).

Цель. Сравнить показатели основного обмена, параметры композиционного состава тела и частоту метаболических нарушений у детей с гипоталамическим и конституционально-экзогенным ожирением.

Memoды. В исследование включены 60 детей в возрасте от 7 до 17 лет с ожирением, разделенных на две группы. В исследуемую группу вошли 20 детей с гипоталамическим ожирением, развившимся после лечения краниофарингиомы. Группу контроля составили 40 детей с конституционально-экзогенным ожирением. Всем детям проведены оценка композиционного состава тела, основного обмена и скрининг метаболических нарушений, ассоциированных с ожирением.

Результаты. Оценка основного обмена выявила значительное снижение данного показателя у детей с гипоталамическим ожирением. Среднее снижение ОО составило 13,1\%, однако у отдельных пациентов достигало 33,4\%. Процентные содержания жировой массы при гипоталамическом и конституционально-экзогенном ожирении значимо не различаются $(39,7 \%$ [36,2; 42,6] vs 38,8\% [35,9; 43,2]; p=0,69). Скрининг метаболических нарушений выявил высокую распространенность метаболических нарушений при гипоталамическом ожирении: нарушение толерантности к глюкозе выявляется в 10\%; дислипидемия - в 55\%, инсулинорезистентность - в 50\%, неалкогольная жировая болезнь печени - в 60\%.

Заключение. Для детей с гипоталамическим ожирением характерно снижение основного обмена, в связи с чем при планировании диетотерапии целесообразно проведение непрямой калориметрии. Гипоталамическое ожирение уже в раннем возрасте ассоциировано с высокой частотой метаболических нарушений и не ассоциировано с более выраженной гиперинсулинемией и инсулинорезистентностью по сравнению с группой конституционально-экзогенного ожирения.

КЛЮЧЕВЫЕ СЛОВА: гИпоталамическое ожирение; краниофарингиома; детское ожирение; основной обмен; композиционный состав тела, инсулинорезистентность.

\title{
COMPARATIVE ASSESSMENT OF ENERGY METABOLISM, BODY COMPOSITION AND METABOLIC FEATURES IN CHILDREN WITH HYPOTHALAMIC AND SIMPLE OBESITY
}

(c) Pavel L. Okorokov*, Alexey L. Kalinin, Natalia A. Strebkova, Maria A. Kareva, Olga V. Vasyukova, Valentina A. Peterkova, Olga B. Bezlepkina

Endocrinology Research Center, Moscow, Russia

BACKGROUND: Hypothalamic obesity often develops after surgical treatment of craniopharyngioma and is characterized by rapid weight gain, high frequency of metabolic disorders, body composition specificity and resistance to standard lifestyle modification approaches and medication therapy of obesity. Recent studies show that one of the mechanisms, explaining weight gain in these children is decrease in resting energy expenditure (REE).

AIMS: To compare REE, body composition parameters, and the frequency of metabolic disorders in children with hypothalamic and simple obesity.

MATERIALS AND METHODS: The study included 60 obese children aged 7 to 17 years, divided into two groups. The study group included 20 children with hypothalamic obesity, developed after craniopharyngioma treatment. The control group consisted of 40 children with simple obesity. Body composition, REE, and metabolic disorders were associated in all children. RESULTS: Children with hypothalamic obesity showed a significant decrease of resting energy expenditure. The average decrease in REE was $13.1 \%$, but in single patients it reached $33.4 \%$. The percentage of fat mass in hypothalamic and simple obesity does not differ significantly $(39.7 \%$ [36.2; 42.6] vs $38.8 \%$ [35.9; 43.2]; $p=0.69)$. Screening for metabolic disorders revealed a high prevalence of metabolic disorders in hypothalamic obesity: impaired glucose tolerance - in 10\%; dyslipidemia - 55\%, insulin resistance-50\%, non-alcoholic fatty liver disease - $60 \%$. 
CONCLUSIONS: Children with hypothalamic obesity showed a significant decrease of resting energy expenditure. When planning a diet in this group of patients it is preferable to use indirect calorimetry. Hypothalamic obesity even at an early age is associated with a high frequency of metabolic disturbance. Hypothalamic obesity in children is not associated with more pronounced hyperinsulinemia and insulin resistance compared to the simple obesity.

KEYWORDS: hypothalamic obesity; craniopharyngioma; pediatric obesity; basal metabolism; body composition, insulin resistance.

\section{ОБОСНОВАНИЕ}

Краниофарингиома (КФ) является наиболее распространенной интракраниальной опухолью у детей, а ее лечение в 55\% случаев сопровождается развитием гипоталамического ожирения (ГО) [1]. При данном типе ожирения стандартные рекомендации по изменению образа жизни и медикаментозная терапия зачастую неэффективны. К основным причинам развития ГО относится повреждение структур гипоталамуса, участвующих в регуляции пищевого поведения, как результат оперативного лечения опухоли. Важная роль в развитии гипоталамического ожирения придается дисфункции вегетативной нервной системы, выражающейся в снижении тонуса симпатической нервной системы, принимающей непосредственное участие в регуляции энергетического обмена в организме [2]. Недавние исследования демонстрируют, что одним из механизмов, объясняющих повышенную прибавку веса у детей после оперативного лечения КФ, является снижение основного обмена (ОO) [3-5].

Гипоталамическое ожирение сопровождается развитием метаболических нарушений, в том числе инсулинорезистентности, уже в раннем детском возрасте. Снижение чувствительности тканей к действию инсулина не только может способствовать прогрессивному набору веса, но и является независимым фактором риска сердечно-сосудистых заболеваний, неалкогольной жировой болезни печени и сахарного диабета 2 типа [6].

Согласно данным литературы, при сравнении композиционного состава тела у детей с гипоталамическим и конституционально-экзогенным ожирением у пациентов с гипоталамическим ожирением отмечается преобладание жировой ткани при относительном уменьшении количества тощей массы. Большинство авторов связывают это с развитием соматотропной недостаточности, выявляемой у 100\% детей после хирургического лечения каринофарингиомы [5, 7].

Таким образом, интересным представляется изучение особенностей энергетического обмена и композиционного состава тела у детей с гипоталамическим ожирением, а также определение частоты инсулинорезистентности (ИР) и других метаболических нарушений в сравнении с группой конституционально-экзогенного ожирения (КЭО).

\section{ЦЕЛЬ}

Сравнить показатели основного обмена, параметры композиционного состава тела и частоту встречаемости метаболических нарушений у детей с гипоталамическим и конституционально-экзогенным ожирением.

\section{МЕТОДЫ}

\section{Дизайн исследования}

Проведено обсервационное одноцентровое одномоментное выборочное неконтролируемое исследование

Критерии соответствия

Критерии включения в основную группу: в группу гипоталамического ожирения включались дети с наличием в анамнезе оперативного лечения КФ в возрасте от 7 до 17 лет.

Критерии включения в контрольную группу: возраст от 7* до 17 лет, конституционально-экзогенное ожирение.

Критерии исключения: другие опухоли ЦНС, кроме КФ (для группы гипоталамического ожирения); декомпенсация по вторичному гипотиреозу, вторичному гипокортицизму, центральному несахарному диабету (для группы гипоталамического ожирения); срок после оперативного лечения КФ не менее 3 месяцев (для группы гипоталамического ожирения); медикаментозная терапия ожирения.

\section{Условия проведения}

и продолжительность исследования

В исследование включались пациенты, находившиеся на стационарном обследовании в ФГБУ «Национальный медицинский исследовательский центр эндокринологии» Минздрава России в период с февраля по июнь 2020 г.

\section{Описание медицинского вмешательства}

Антропометрические измерения включали в себя: измерение роста, веса, расчет индекса массы тела (ИМТ). ИМТ оценивался по нормативам для конкретного возраста и пола и представлен в виде числа стандартных отклонений от среднего (SDS). Всем пациентам проводилась оценка полового развития, композиционного состава тела и исследование уровня основного обмена. Биохимическое исследование крови включало определение общего холестерина, холестерина липопротеидов низкой плотности (ЛПВП), холестерина липопротеидов высокой плотности (ЛПВП), триглицеридов, аланинаминотрансферазы (АЛТ). Также всем пациентам проводился стандартный пероральный глюкозотолерантный тест (ПГТТ). Для оценки ИР использовался индекс HOMA-IR. Выбор HOMAIR как косвенного критерия ИР продиктован необходимостью сравнения полученных результатов с данными других исследований, в подавляющем большинстве которых для оценки ИР при гипоталамическом ожирении использовался именно этот показатель. Исследование уровня инсулиноподобного фактора роста 1 (ИФР-1) и расчет SDS ИФР-1 проведен всем пациентам с ГО и 29 пациентам из группы КЭО.

\footnotetext{
* В связи с техническими сложностями при проведении респираторной калориметрии.
} 


\section{Основной исход исследования}

В качестве основных конечных точек исследования были приняты следующие параметры: абсолютные значения ОО, значения ОО с поправкой на тощую массу (ОО/ТМ), интенсивность ОО (сниженный, нормальный или повышенный ОО), процентное содержание жировой ткани для их последующего сравнения в исследуемых подгруппах КЭО и ГО.

\section{Дополнительные исходы исследования}

В качестве дополнительных конечных точек исследования были приняты параметры, характеризующие частоту метаболических нарушений: дислипидемии, нарушений углеводного обмена, ИР и неалкогольной жировой болезни печени (НАЖБП) в исследуемых подгруппах.

\section{Анализ в подгруппах}

Всех участников исследования разделили на две подгруппы: подгруппу с ГО, куда включали детей с ожирением, развившимся после оперативного лечения КФ, и подгруппу сравнения, включавшую детей с КЭО.

\section{Методы регистрации исходов}

Ожирение диагностировалось при значении SDS ИMT $\geq 2,0$, согласно национальным клиническим рекомендациям по диагностике и лечению ожирения, основанным на нормативах Всемирной организации здравоохранения (ВО3). Оценка полового развития проводилась по классификации Tanner. Биохимические исследования выполняли на автоматическом анализаторе Architect c8000 (Abbott Laboratories). Дислипидемия диагностировалась при повышении уровня общего холестерина $\geq 5,2$ ммоль/л или повышении уровня триглицеридов >1,3 ммоль/л (для детей до 10 лет); $\geq 1,7$ ммоль/л (для детей старше 10 лет). Диагноз НАЖБП устанавливался при повышении АЛТ >25 Ед/л для мальчиков и >22 Ед/л - для девочек [8]. Состояние углеводного обмена оценивали по результатам ОГТТ с глюкозой из расчета 1,75 г/кг, но не более 75 г сухого вещества на основании рекомендаций ВОЗ (1998). Нормальной считалась концентрация глюкозы в венозной плазме натощак <6,1 ммоль/л; глюкозы венозной плазмы на 120-й мин ОГТТ <7,8 ммоль/л. Уровень инсулина определялся на электрохемилюминесцентном анализаторе Cobas 6000 (Roche). За критерий ИР принято значение индекca Homeostasis Model Assessment of Insulin Resistance (HOMA-IR) $\geq 2,5$ [7]. Оценка композиционного состава тела проводилась методом биоимпедансного анализа (анализатор Tanita BC-418, Япония) утром, натощак. Измерение основного обмена проводилось методом непрямой респираторной калориметрии на метаболоrрафе Quark RMR (Cosmed, Италия). Исследование выполнялось утром, натощак, в условиях покоя и температурного комфорта (температура в помещении 22-26 C), в течение 15 минут в положении пациента лежа. Данные, полученные в первые 5 минут, исключались из последующего анализа, а оценка ОО проводилась по достижении устойчивого состояния не менее 10 минут. Долженствующее значение основного обмена рассчитывалось по формуле Harris-Benedict (1919 г.). Снижение и повышение интенсивности основного обмена определялось при отклонении фактического (измеренного методом респираторной калориметрии) значения ОО от долженствующего > $>10 \%$ соответственно. В остальных случаях скорость ОО расценивалась как нормальная.

\section{Этическая экспертиза}

Протокол исследования одобрен 12.02.2020 г. локальным этическим комитетом при ФГБУ «Национальный медицинский исследовательский центр эндокринологии» Минздрава России (выписка из протокола №2 от 12.02.2020 г.).

\section{Статистический анализ}

Принципы расчета размера выборки: размер выборки предварительно не рассчитывался.

Методы статистического анализа данных. Статистическая обработка данных проводилась с использованием пакета прикладных программ Statistica (StatSoftlnc., USA, version 10.0). Так как большинство изучаемых показателей не имело приближенно-нормального распределения, все данные представлены в виде медианы и интерквартильного размаха. Для оценки достоверности различий между изучаемыми подгруппами использовался критерий Манна-Уитни и дисперсионный анализ Краскела-Уоллеса. Корреляционный анализ проводился с использованием критерия Спирмена. Критический уровень значимости различий принимали $\leq 0,05$.

\section{РЕЗУЛЬТАТЫ}

Объекты (участники) исследования

Всего в исследование включены 60 детей (25 мальчиков, 35 девочек) с ожирением (SDS ИМТ 2,75 [2,3; 2,9]) в возрасте от 7 до 17 лет (средний возраст 12 лет [9,8; 14]). В подгруппу гипоталамического ожирения вошли 20 детей (8 мальчиков, 12 девочек) с ожирением (SDS ИМТ $2,4[2,1 ; 3,1])$. Подгруппу сравнения составили 40 детей (17 мальчиков, 23 девочки) с КЭО (SDS ИМТ 2,7 [2,4; 2,9]). Клинические характеристики подгрупп представлены в Табл. 1.

Таблица 1. Клиническая характеристика исследуемых групп.

\begin{tabular}{lccc}
\hline & ГО & KЭO & p \\
\hline Количество пациентов, $\mathrm{n}$ & 20 & 40 & NS \\
Пол (\% девочек) & 60 & 57,5 & NS \\
Возраст, лет & $10,5[8,5 ; 14,5]$ & $11,5[10,1 ; 13,6]$ & 0,0001 \\
SDS роста & $-0,51[-1,5 ;-0,89]$ & $1,46[0,1 ; 2,5]$ & NS \\
SDS ИМT & $2,4[2,1 ; 3,1]$ & $2,7[2,4 ; 2,9]$ & \\
\hline
\end{tabular}

Примечания. ГО - гипоталамическое ожирение; КЭО - конституционально-экзогенное ожирение. Данные представлены В виде медианы с интерквартильным интервалом Me [Q1;Q3]. SDS - стандартное отклонение от среднего. NS - not significant (не значимо). 
Исследуемая и контрольная подгруппы были сопоставимы по возрасту, полу и SDS ИМТ, однако значимо различались по SDS роста ( $p=0,0001)$ (см. табл. 1). В подгруппе ГО преобладали допубертатные пациенты (1 стадия по Таннеру определяется у $60 \%, 2-3$ стадия - у $25 \%$, 4-5 стадия - у 15\%), в то время как при КЭО доля детей с 1 стадией полового развития по Таннеру составила лишь 27,5\%.

В подгруппе ГО соматотропная недостаточность диагностирована у всех детей. Вторичный гипокортицизм и вторичный гипотиреоз выявлены в 80 и $85 \%$ случаев соответственно. Центральный несахарный диабет диагностирован в 95\% случаев. Вторичный гипогонадизм выявлен у одного пациента. Все гормональные недостаточности, за исключением СТГ-дефицита, были медикаментозно компенсированы. Ни один пациент с ГО и соматотропной недостаточностью на момент исследования не получал терапию гормоном роста.

\section{Основные результаты исследования}

Исследование параметров композиционного состава тела не выявило статистически значимых различий в содержании жировой ткани в подгруппах гипоталамического и КЭО (39,7\% [36,2; 42,6] vs 38,8 \% [35,9; 43,2] соответственно; $p=0,69)$. Количество тощей массы у детей с КЭО было значительно больше, по сравнению с группой ГО $(41,7$ кг $[36,3 ; 49,0]$ vs 31,5 кг $[22,8 ; 42,1]$ соответственно; $p=0,0001)$.Оценка энергетического обмена выявила значительное снижение основного обмена в подгруппе гипоталамического ожирения. Средние значения ОО составили 1181 [1027; 1519] и 1756 [1505; 1852] ккал/сутки для подгрупп гипоталамического и конституционально-экзогенного ожирения, соответственно ( $p=0,0001)$. Снижение скорости

Рисунок 1. Интенсивность основного обмена в группах гипоталамического и конституционально-экзогенного ожирения

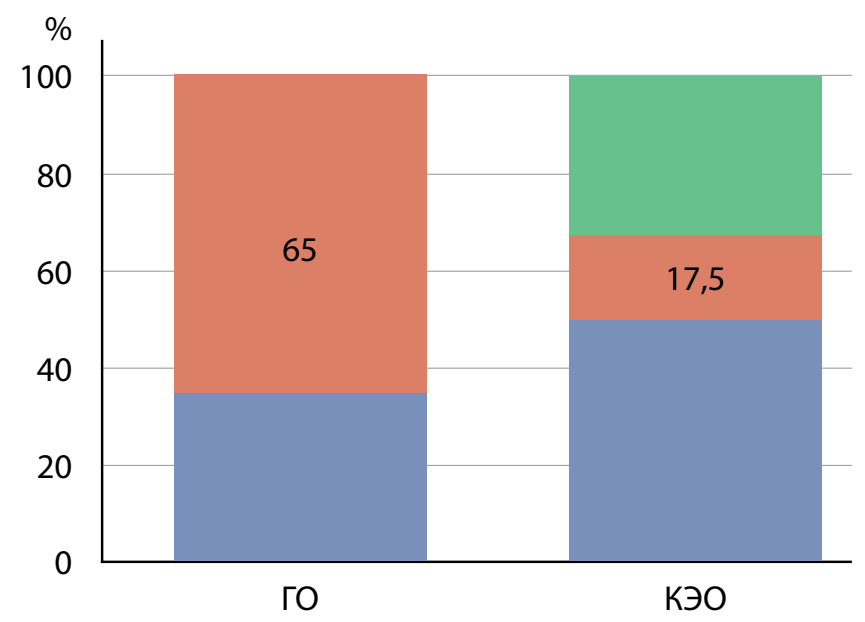

ОО повышен

ОО снижен

ОО норма

Примечание. ГО - гипоталамическое ожирение, КЭО - конституционально-экзогенное ожирение, ОО - основной обмен базального метаболизма выявлено у 65\% детей с гипоталамическим ожирением и лишь у 17,5\% детей с простым ожирением (см. рис. 1). Нормальные показатели основного обмена определялись в 35 и 50\% случаев, соответственно. Повышение скорости энергообмена в покое вообще не зафиксировано при ГО и встречается у $32,5 \%$ детей из подгруппы конституционально-экзогенного ожирения. Среднее снижение основного обмена в подгруппе гипоталамического ожирения составило $13,1 \%$, однако у отдельных пациентов достигало $33,4 \%$.

При проведении корреляционного анализа продемонстрирована положительная взаимосвязь основного обмена с количеством тощей массы, как в группе гипоталамического, так и конституционально-экзогенного ожирения ( $r=0,83$ и $r=0,5$, соответственно; $p<0,05)$. Степень ожирения (SDS ИMT) и количество жировой ткани в организме (\% жира) не оказывают существенного влияния на показатели энергообмена в покое. Учитывая, что тощая масса оказывает наибольшее влияние на основной обмен, дальнейший анализ проводился с поправкой на нее (основной обмен, ккал / тощая масса, кг - ОО/ТМ). При оценке основного обмена в группах гипоталамического и конституционально-экзогенного ожирения с поправкой на тощую массу значимых различий между группами выявлено не было ( сохраняется тенденция к снижению ОО в группе гипоталамического ожирения.Корреляционный анализ выявил положительную взаимосвязь ОО/TM с SDS ИФР-1 ( $r=0,64$; $\mathrm{p}<0,05)$, но лишь в группе гипоталамического ожирения. Среднее значение SDS ИФР-1 в подгруппе гипоталамического ожирения составило -1,2 [-3,5; -0,1], а в подгруппе КЭО -0,7 [-1,3; 0,2]; исследуемые показатели значимо различались $(p=0,04)$

Рисунок 2. Значения основного обмена с поправкой на тощую массу при конституционально-экзогенном и гипоталамическом ожирении

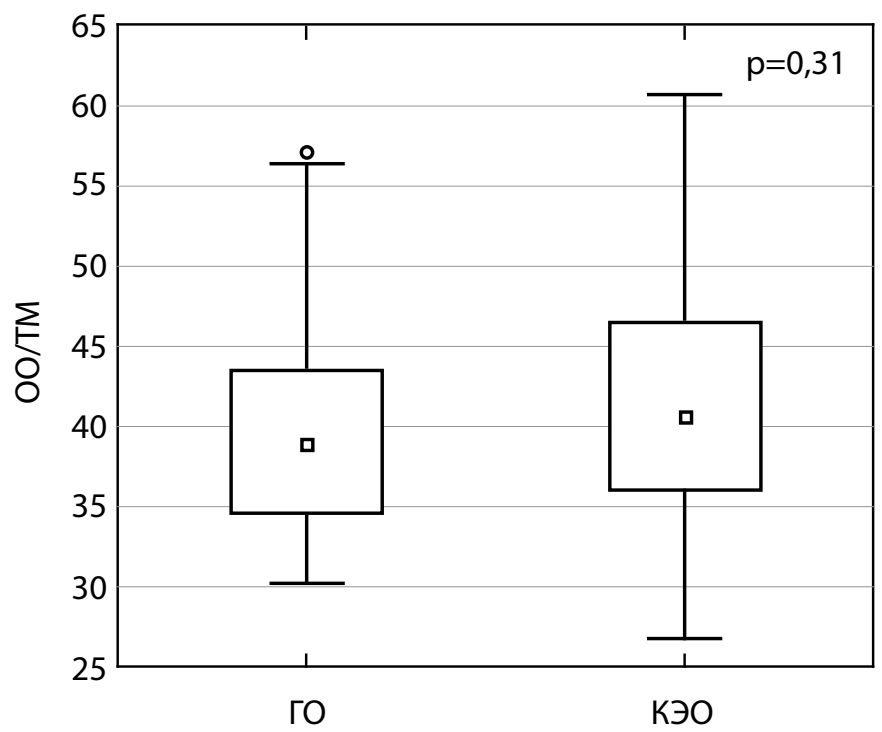

Примечание. ГО - гипоталамическое ожирение, ОО/ТМ - отношение основного обмена к тощей массе, ккал/кг. 
Таблица 2. Показатели метаболического профиля в исследуемых группах.

\begin{tabular}{lccc}
\hline & ГО & KЭО & p \\
\hline Общий холестерин, ммоль/л & $4,4[3,8 ; 5,7]$ & $4,4[3,8 ; 5,1]$ & NS \\
Триглицериды, ммоль/л & $1,5[1,1 ; 2,1]$ & $1,1[0,9 ; 1,4]$ & 0,01 \\
АЛТ, Ед/л & $24[17 ; 29]$ & $19[16 ; 23]$ & NS \\
Глюкоза 0 мин., ммоль/л & $4,1[3,8 ; 4,5]$ & $4,7[4,5 ; 4,9]$ & NS \\
Глюкоза 120 мин, ммоль/л & $6,2[5,8 ; 7,1]$ & $5,9[5,6 ; 6,4]$ & NS \\
Инсулин, мкЕд/л & $17,1[10,4 ; 22,4]$ & $18,5[11,7 ; 25,7]$ & NS \\
НОМА-IR & $3,1[1 ; 4,5]$ & $3,9[3,1 ; 5,5]$ & 0,01
\end{tabular}

Примечания. ГО - гипоталамическое ожирение, КЭО - конституционально-экзогенное ожирение.

Данные представлены в виде медианы с интерквартильным интервалом Me [Q1; Q3]. HOMA-IR - индексинсулинорезистентности (Нотеоstasis Model Assessment of Insulin Resistance)

\section{Дополнительные результаты исследования}

Скрининг метаболических нарушений выявил высокую распространенность ассоциированных с ожирением состояний как при конституционально-экзогенном, так и при гипоталамическом ожирении. Нарушение толерантности к глюкозе выявляется в обеих исследуемых подгруппах с сопоставимой частотой (10\% vs 7,5\%). Дислипидемия при ГО диагностирована у 11 детей (55\%) и встречается значительно чаще по сравнению с подгруппой КЭО (37,5\%). Обращают на себя внимание высокие показатели триглицеридов сыворотки в подгруппе гипоталамического ожирения (см. табл. 2). Частота инсулинорезистентности в подгруппе гипоталамического ожирения составила 50\%, в подгруппе КЭО - 95\% случаев (38 из 40 детей). Так, значение индекса HOMA-IR в группе ГО составило 3,1 [1; 4,5], а при КЭО - 3,9 [3,1; 5,5] (p=0,01). Неалкогольная жировая болезнь печени с большей частотой встречается при гипоталамическом ожирении в сравнении с подгруппой КЭО (60 vs 25\%).

\section{Нежелательные явления}

В ходе проведения исследования нежелательных явлений не зафиксировано. Оценка композиционного состава тела и исследование основного обмена являются неинвазивными диагностическими методами, а их проведение не сопровождалось ухудшением самочувствия или другими жалобами.

\section{ОБСУЖДЕНИЕ}

\section{Резюме основного результата исследования}

Оценка особенностей энергетического обмена продемонстрировалазначительное снижение интенсивности основного обмена у детей с гипоталамическим ожирением, выявляемое у 65\% пациентов. \% содержание жировой ткани при гипоталамическом и конституционально-экзогенном ожирении значимо не различались. Однако выявлено увеличение абсолютного количества тощей массы у детей с конституционально-экзогенным ожирением. SDS ИМТ и \% содержание жировой ткани в организме не оказывают существенного влияния на снижение интенсивности основного обмена при различных формах ожирения.

Обсуждение основного результата исследования

Выявленное в настоящем исследовании снижение скорости основного обмена у детей с ожирением, раз- вившемся после оперативного лечения КФ, продемонстрировано и в ряде других работ $[4,5,7]$. В исследовании Ilanit Bommer c соавт. в группе детей после оперативного лечения краниофарингиомы, выявлено значимое снижение основного обмена по сравнению с контрольной группой (43 ребенка в конституционально-экзогенным ожирением), сопоставимой с исследуемой группой по полу, возрасту и SDS ИМТ (1297 vs 1721 ккал; $р<0,01)$. Данные различия сохранялись и после поправки на тощую массу, чего не выявлено в нашем исследовании [7]. В работе M. Guftar Shaikh с соавт. также определяется снижение основного обмена в группе из 18 детей с гипоталамическим ожирением (средний возраст 13,5 лет, средний SDS ИMT +2,74) [4]. Авторы также отмечают снижение двигательной активности в исследуемой группе при отсутствии значимого повышения аппетита, и приходят к выводу, что снижение ОО и двигательной активности являются более значимыми факторами прибавки веса при гипоталамическом ожирении, чем количество потребляемой пищи. В исследовании, включившем 11 пациентов с КФ в анамнезе, из которых 8 имели ожирение, выявлено снижение ОО в группе гипоталамического ожирения в среднем на 17\% [5]. В нашей работе среднее снижение основного обмена в группе гипоталамического ожирения составило $13,1 \%$, однако у отдельных пациентов достигало 33,4\%, в связи с чем, при планировании диетотерапии для лечения гипоталамического ожирения у детей целесообразно проведение непрямой калориметрии.

Снижение основного обмена было выявлено нами у 65\% детей с гипоталамическим ожирением, и у 7,5\% при конституционально-экзогенном ожирении. В других работах в также отмечается значимое снижение энергозатрат в покое у детей с КФ в анамнезе (у 77\%) по сравнению с группой КЭО (у 30\%) [7]. При сравнительном анализе выявлена тенденция к увеличению SDSИMT и \% содержания жировой ткани в организме у детей с гипоталамическим ожирением и сниженным основным обменом, не достигшая статической значимости (данные не представлены). Исследуемые подгруппы были сопоставимы по метаболическим показателям. Для уточнения влияния интенсивности основного обмена на выраженность ожирения, композиционный состав тела и развитие метаболических нарушений необходимы дальнейшие исследования с увеличением размеров выборки детей с гипоталамическим ожирением. Также 
интересным направлением для изученияявляется определение предиктивной роли снижения основного обмена в прогрессировании гипоталамического ожирения для чего планируется продолжить динамическое наблюдение за включенными в данное исследование пациентами.

Известно, что для планирования суточного рациона питания необходимо знание уровня основного обмена. Для его определения используются расчетные формулы или метод непрямой респираторной калориметрии, являющийся «золотым стандартом» для оценки основного обмена. В нашем исследовании показано, что у детей с гипоталамическим ожирением использование расчетной формулы (Harris-Benedict, 1919 г.) в 65\% случае переоценивает суточные энергозатраты в покое, что неизбежно приводит к формированию «избыточного» по калорийности рациона питания и снижению эффективности диетотерапии. В этой связи, использование непрямой калориметрии у лиц с гипоталамическим ожирением дает определенные преимущества, т.к. позволяет учитывать индивидуальные потребности ребенка в энергии.

Согласно данным литературы, именно тощая масса является основным фактором вариабельности основного обмена как при конституционально-экзогенном [9], так и при гипоталамическом ожирении [7]. В нашем исследовании также выявлена сильная положительная корреляционная взаимосвязь показателей основного обмена с количеством тощей массы в группах гипоталамического и конституционально-экзогенного ожирения. Не продемонстрировано значимого влияния количества жировой массы в организме и SDS ИMT на уровень энергообмена в покое. Оценка особенностей композиционного состава тела при гипоталамическом и конституционально-экзогенном ожирении в нашей работе не выявила значимых различий. Однако большинство авторов демонстрируют более высокое содержание жировой ткани и уменьшение количества тощей массы у детей с гипоталамическом ожирением, по сравнению с КЭО [5, 7]. Данный факт авторы объясняют дефицитом тестостерона и соматотропного гормона у лиц гипоталамическим ожирением. Отсутствие различий в параметрах композиционного состава тела в нашей работе может быть связано с тем, что средний возраст детей, включенных в исследование составил 12 лет и большинство из них имело допубератное половое развитие, что нивелирует анаболическое влияние половых стероидов на количество скелетной мышечной массы.

Среди других факторов, оказывающих влияние на скорость основного обмена при гипоталамическом ожирении, рассматриваются тиреоидные и половые гормоны, а также инсулиноподобный фактор роста-1. В проведенном нами исследовании выявлена положительная взаимосвязь основного обмена с поправкой на тощую массу и SDS ИФР-1, что может свидетельствовать о влиянии дефицита гормона роста на энергетический обмен в покое независимо от количества тощей массы. Так как пациенты с гипоталамическим ожирением не получали заместительную терапию рекомбинантным гормоном роста при планировании дальнейших исследований представляется интересным изучение влияния данной терапии на интенсивность основного обмена, содержа- ние тощей массы в организме и возможность предотвращения дальнейшей прогрессии ожирения. Оценка половых гормонов большинству пациентов, включенных в исследование, не проводилась в связи с возрастом и стадией полового развития, соответствующего допубертатным значениям по шкале Таннера.

В работе M. Guftar Shaikh с соавт. выявлена положительная корреляционная взаимосвязь свободного Т3 (но не свободного Т4) с показателями основного обмена $(r=0,46 ; p<0,01)$ у подростков с гипоталамическим ожирением [4]. В нашем исследовании оценка уровня свободного Т3 не проводилось.

Согласно современным представлениям, повреждение гипоталамуса при инвазивном росте КФ или в ходе оперативного лечения, может приводить к формированию ИР. Обусловлено это нарушением а- MSH сигналинга, которое приводит к повышению тонуса блуждающего нерва, нарушению чувствительности к инсулину гипоталамических структур и развитию компенсаторной гиперинсулинемии [6].

В нашем исследовании достоверных различий В уровнях инсулина натощак между подгруппами ГО и КЭО не выявлено $(17,1$ [10,4-22,4] vs 18,5 [11,7-25,7]; $\mathrm{p}=0,56)$. В группе детей с конституционально-экзогенным ожирением выявлена более высокая частота ИР (95\%) по сравнению со сверстниками после оперативного лечения КФ (50\%) при сопоставимых значениях SDS ИМT. Полученные результаты согласуются с данными других авторов, также не выявивших более выраженной гиперинсулинемии и ИР у детей с ГО по сравнению с КЭО [3, 7, 10]. В работе Guran T. С соавт., включившей 23 ребенка с гипоталамическим ожирением (средний возраст 10,3 лет; средний SDS ИMT +2,0) и группу контроля из 22 детей с простым ожирением, уровни инсулина натощак в группах составили 16 мЕд/л и 28 мЕд/л, соответственно. По индексу HOMA-IR дети с гипоталамическим и конституционально-экзогенным ожирением также значимо различались (2,8 и 6,5 соответственно $(p<0,05))$, причем большей степенью инсулинорезистентности характеризовалась именно группа КЭО. В работе IlanitBommer с соавт. вообще найдено значимых различий в индексе HOMA-IR между группами конституционально-экзогенного и гипоталамического ожирения $(2,39$ [0,2-13,9] vs 3,2 [0,9-10,9]) [7]. Противоречивые данные по частоте и выраженности ИР связаны с отсутствием единых диагностических критериев в детском возрасте, в том числе и при гипоталамическом ожирении. Однако в большинстве работ по исследованию метаболических нарушений при ГО у детей именно индекс HOMA-IR был выбран в качестве критерия ИР. Повышение уровней сывороточных триглицеридов и высокая частота неалкогольной жировой болезни печени (НАЖБП), продемонстрированные в нашем исследовании, также выявляют и другие авторы $[11,12]$. В работе Anika Hoffmann с соавт., НАЖБП выявлена у 50\% пациентов после лечения КФ, что сопоставимо с полученной в нашем исследовании частотой в $60 \%$ [11].

Таким образом, у детей сгипоталамическим ожирением уже в возрасте 10-11 лет отмечается высокая частота различных метаболических нарушений, даже несмотря на незначительную выраженность ожирения (средний SDS ИMT +2,4). 


\section{Ограничения исследования}

К основному недостатку настоящего исследования следует отнести небольшой объем выборки детей с гипоталамическим ожирением.

\section{ЗАКЛЮЧЕНИЕ}

Снижение энергетического обмена при гипоталамическом ожирении ранее было описано рядомавторов. В нашем исследование среднее снижение энергозатрат покоя при гипоталамическом ожирении, развившимся вследствие оперативного лечения КФ составило 13,1\%, однако у отдельных пациентов достигало 33,4\%. Выявленные изменения ОО способствуют формированию «положительного» энергетического баланса и могут объяснять прогрессивный набора веса у данных пациентов. Планировании диетотерапии с учетом особенностей энергетического обмена у детей с гипоталамическим ожирением может способствовать повышению эффективности программ по снижению веса. У детей с гипоталамическим ожирением уже в раннем возрасте определяется высокая частота метаболических нарушений, таких как ИР, дислипидемия, и неалкогольной жировой болезни печени. В этой связи целесообразно более раннее проведение скрининга метаболических нарушений в данной группе пациентов. Полученные результаты открывают возможности для продолжения исследований, направленных на выявление других факторов, приводящих к снижению основного обмена при гипоталамическом ожирении и оценку предиктивной роли снижения интенсивности ОО на развитие и течение ожирения в будущем. Перспективным направлением для дальнейших исследований также является оценка влияния терапии гормоном роста на показатели композиционного состава тела, энергетического обмена и частоту метаболических нарушений при гипоталамическом ожирении.

\section{ДОПОЛНИТЕЛЬНАЯ ИНФОРМАЦИЯ}

Источники финансирования. Исследование выполнено в рамках НИР: «Новые подходы к персонифицированному лечению ожирения у детей на основе исследований энергетического обмена, функционального резерва бета-клеток, секреции адипокинов, миокинов и специфических шаперонов», регистрационный номер AAAA-A20-120011790172-9.

Конфликт интересов. Авторы декларируют отсутствие явных и потенциальных конфликтов интересов, связанных с содержанием настоящей статьи

Участие авторов. Окороков П.Л. - разработка протокола исследования, сбор материала, проведение непрямой респираторной калориметрии, обработка и интерпретация результатов, подготовка рукописи; Калинин А.Л. - сбор и обработка материала, интерпретация результатов, подготовка рукописи; Стребкова Н.А. - разработка протокола исследования, интерпретация результатов, подготовка рукописи; Карева М.А. - контроль и координация проведения исследования, критическая интерпретация результатов, написание текста; Васюкова О.В. - сбор и обработка материалов, проведение непрямой респираторной калориметрии, критическая интерпретация результатов; Петеркова В.А. разработка и утверждение протокола исследования, критическая интерпретация результатов, написание текста; Безлепкина О.Б. - разработка и утверждение протокола исследования, критическая интерпретация результатов, написание текста. Все авторы одобрили финальную версию статьи перед публикацией, выразили согласие нести ответственность за все аспекты работы, подразумевающую надлежащее изучение и решение вопросов, связанных с точностью или добросовестностью любой части работы.

\section{СПИСОК ЛИТЕРАТУРЫ | REFERENCES}

1. Müller HL, Bueb K, Bartels U, et al. Obesity after childhood craniopharyngioma--German multicenter study on pre-operative risk factors and quality of life. Klin Padiatr. 2001;213(4):244-249. doi: https://doi.org/10.1055/s-2001-16855

2. Roth C, Hunneman D, Gebhardt U, et al. Reduced Sympathetic Metabolites in Urine of Obese Patients with Craniopharyngioma. Pediatr Res. 2007;61 (4):496-501. doi: https://doi.org/10.1203/pdr.0b013e3180332cd6

3. Simoneau-Roy J, O'Gorman C, Pencharz P, et al. Insulin sensitivity and secretion in children and adolescents with hypothalamic obesity following treatment for craniopharyngioma. Clin Endocrinol (Oxf). 2010;72(3):364-370. doi: https://doi.org/10.1111/j.1365-2265.2009.03639.x

4. Shaikh MG, Grundy RG, Kirk JM. Reductions in basal metabolic rate and physical activity contribute to hypothalamic obesity. J Clin Endocrinol Metab. 2008;93(7):2588-2593. doi: https://doi.org/10.1210/jc.2007-2672

5. Kim R, Shah R, Tershakovec A, et al. Energy expenditure in obesity associated with craniopharyngioma. Childs Nerv Syst. 2010;26(7):913-917. doi: https://doi.org/10.1007/s00381-009-1078-1

6. Lustig RH. Autonomic Dysfunction of the $\beta$-Cell and the Pathogenesis of Obesity. Rev Endocr Metab Disord. 2003;4(1):23-32. doi: https://doi.org/10.1023/A:1021819318484

7. Bomer I, Saure C, Caminiti C, et al. Comparison of energy expenditure, body composition, metabolic disorders, and energy intake between obese children with a history of craniopharyngioma and children with multifactorial obesity. J Pediatr Endocrinol Metab. 2015;28(11-12):1305-1312. doi: https://doi.org/10.1515/jpem-2015-0167

8. Styne DM, Arslanian SA, Connor EL, et al. Pediatric ObesityAssessment, Treatment, and Prevention: An Endocrine Society Clinical Practice Guideline. J Clin Endocrinol Metab. 2017;102(3):709-757. doi: https://doi.org/10.1210/jc.2016-2573

9. Окороков П.Л., Васюкова О.В., Ширяева Т.Ю. Скорость основного обмена в покое и факторы его вариабельности у подростков с простым ожирением // Вопросы детской диетологии. - 2019. T. 17. - №3. - C. 5-9. [Okorokov PL, Vasyukova OV, Shiryaeva TY. Resting metabolic rate and factors of its variability in adolescents with obesity. Problems of pediatric nutritiology. 2019;17(3)5-9. (In Russ.)] doi: https://doi.org/10.20953/1727-5784-2019-3-5-9

10. Guran T, Turan S, Bereket A, et al. The role of leptin, soluble leptin receptor, resistin, and insulin secretory dynamics in the pathogenesis of hypothalamic obesity in children. Eur J Pediatr. 2009;168(9):1043-1048. doi: https://doi.org/10.1007/s00431-008-0876-x

11. Hoffmann A, Bootsveld K, Gebhardt U, et al. Nonalcoholic fatty liver disease and fatigue in long-term survivors of childhoodonset craniopharyngioma. Eur J Endocrinol. 2015;173(3):389-397. doi: https://doi.org/10.1530/EJE-15-0422

12. Srinivasan $S$, Ogle GD, Garnett SP, et al. Features of the metabolic syndrome after childhood craniopharyngioma. J Clin Endocrinol Metab. 2004;89(1):81-86. doi: https://doi.org/10.1210/jc.2003-030442 
ИНФОРМАЦИЯ ОБ АВТОРАХ [AUTHORS INFO]:

* Окороков Павел Леонидович, к.M.H. [Pavel L. Okorokov, MD, PhD]; адрес: Россия, 117036, Москва, ул. Дмитрия Ульянова, д. 11 [address: 11 Dm. Ulyanova street, 117036, Moscow, Russia];

ORCID: https://orcid.org/0000-0001-9834-727X; eLibrary SPIN: 6989-2620; e-mail: pokorokov@gmail.com

Калинин Алексей Леонидович [Alexey L. Kalinin, MD]; ORCID: https://orcid.org/0000-0003-4142-4355;

eLibrary SPIN: 3543-7179; e-mail: kalinin.sh@gmail.com

Стребкова Наталья Анатольевна, к.м.н. [Natalia A. Strebkova, MD, PhD];

ORCID: https://orcid.org/0000-0002-2410-53-47; eLibrary SPIN: 9897-4858; e-mail: strebcovanata@gmail.com

Карева Мария Андреевна, д.м.н. [Maria A. Kareva, MD, PhD]; ORCID: http://orcid.org/0000-0003-1320-6561;

eLibrary SPIN: 5089-0310; e-mail: i_marusya@mail.ru

Васюкова Ольга Владимировна, к.м.н. [Olga V. Vasyukova, MD, PhD]; ORCID: https://orcid.org/0000-0002-9299-1053;

eLibrary SPIN: 6432-3934; e-mail: o.vasyukova@mail.ru

Петеркова Валентина Александровна, д.м.н., професcop [Valentina A. Peterkova, MD, PhD, professor];

ORCID: https://orcid.org/0000-0002-5507-4627; eLibrary SPIN: 4009-2463; e-mail: peterkovava@hotmail.com

Безлепкина Ольга Борисовна, д.м.н. [Olga B. Bezleokina, MD, PhD]; ORCID: https://orcid.org/0000-0001-9621-5732;

eLibrary SPIN: 3884-0945; e-mail: olgabezlepkina@mail.ru

*Автор, ответственный за переписку / Corresponding author.

\section{ЦИТИРОВАТЬ:}

Окороков П.Л., Калинин А.Л., Стребкова Н.А., Карева М.А., Васюкова О.В., Петеркова В.А., Безлепкина О.Б. Сравнительная оценка энергетического обмена, особенностей композиционного состава тела и метаболических нарушений у детей с гипоталамическим и конституционально-экзогенным ожирением // Ожирение и метаболизм. - 2020. - Т. 17. - №3. C. 249-256. doi: https://doi.org/10.14341/omet12552

\section{TO CITE THIS ARTICLE:}

Okorokov PL, Kalinin AL, Strebkova NA, Kareva MA, Vasyukova OV, Peterkova VA, Bezlepkina OB. Comparative assessment of energy metabolism, body composition and metabolic features in children with hypothalamic and simple obesity. Obesity and metabolism. 2020;17(3):249-256. doi: https://doi.org/10.14341/omet12552 